\title{
Thermal Design of High Performance Magneto-Optical Data Storage Films
}

\author{
T.W. McDaniel \\ Quinta Corporation, San Jose, California 95131-1826, USA
}

\begin{abstract}
Thermal design issues for magneto-optical media are reviewed. New simulation results for thermomagnetic recording, particularly under high speed, high density conditions, are presented. Achievement of high areal density recording through use of thermal confinement features is discussed. Micromagnetic recording simulations done in the presence of heat flow control measures are provided.
\end{abstract}

Key words: magneto-optical (MO) recording, MO thermal design, film design

\section{Introduction}

Development of rewritable magneto-optical (MO) media has been advancing rapidly since the introduction of amorphous rare-earth transition metal (RE-TM) alloys in the 1970's. Products using MO disks are now shipping with areal densities in the range of approximately $2-6$ Gbit/ $\mathrm{in}^{2}$, and advanced designs have been reported ${ }^{1)-3}$ ) which promise to extend this level from one to perhaps two orders of magnitude. Not only are the thin film designs evolving, but recently several organizations have disclosed new storage device architectures which will utilize MO media in new ways for high performance (areal density and data rate) recording 2),3). These developments are increasing the criticality of proper media design (optical, thermal, magnetic) in order to meet the demands of an expanding data storage user base.

Both the recording and readback processes in MO data storage rely on the thermal properties of the thin film coatings on the disk as they are integrated with the substrate. In general, as new high performance MO applications are introduced, the task of the media design engineer is to assure that smaller magnetic domains can be reliably placed and detected at ever higher rates. The recent introduction of applications which combine architectural aspects of magnetic hard disk drives (HDD's) with those of optical disk drives (ODD's) means that $\mathrm{MO}$ disks will be spun at higher rates, and that flying heads will be used with first-surface film structures ${ }^{2,3)}$. An important change for the designer is that the relative linear speeds at which MO media are used will increase into new regimes.

In this paper, we will recall some of the established fundamentals of the thermal behavior of media in $\mathrm{MO}$ recording that should guide our analysis, and then consider some of the novel issues that arise in high rate, high density thermomagnetic recording and MO readout. We will explore some of the media thermal design strategies and techniques that may be useful for future MO disks.

\section{Media Thermal Engineering Fundamentals}

Much has been published on thermal performance of optical media ${ }^{4)}$-7). We begin by pointing out a few simple, but very useful behaviors that often have not been made explicit in earlier work. We should point out that the scope of the engineering analysis in the references cited, as well as in the present work, is well within the limits of classical heat conduction in homogeneous media. This means that the well-known analysis of classical references ${ }^{8}$ applies, and we need not concern ourselves with the complications of microscale heat transport ${ }^{4) .9)}$ or phase transitions. Although these are helpful simplifications for the analysis, we still must (like previous workers) resort to numerical analyses of heat conduction by computer due to the somewhat complex thin film and disk substrate structures in which we are interested. Non-numerical thermal analysis methods for multilayer thin film stacks, even those on planar substrates ${ }^{10)}$, are just too complicated for routine engineering design practice.

Probably the most important fundamental to remember in the heating of moving thin film structures with focused light beams is the following: The most efficient heating with the highest resultant thermal gradients is always achieved through the use of short duration irradiation pulses. This follows from the experimental fact that heat conduction, particularly in solids, obeys a diffusion-like differential equation upon which all of the analysis of $\mathrm{MO}$ recording can be based:

$$
\begin{aligned}
& \nabla^{2} T-\frac{1}{\kappa} \frac{\partial T}{\partial t}=\frac{Q(x, y, z, t)}{k}=A \cdot \exp \left[-\beta^{2}\left(\{x-v t\}^{2}+y^{2}\right)\right] \\
& \quad \exp (-\alpha z) \Psi(t)
\end{aligned}
$$

where $T(x, y, z, t)$ is the temperature field of interest, $k=k / \rho c$ is the thermal diffusivity, $k$ is the thermal conductivity, $\rho$ is the material mass density, $c$ is the material mass specific heat, $v$ is the moving media velocity, and $Q$ is the heat energy input. In the case of laser beam heating for recording, $\mathrm{Q}$ is often represented as the Poynting vector energy deposition rate from absorption of a Gaussian profile electromagnetic beam according to these parameters:

- $\alpha$ is a Beer's Law absorption coefficient;

- $\beta$ is an inverse beam size parameter;

- $\Psi(t)$ is a beam power versus time profile.

In practice, a numerical solution to (1) will usually have a provision to accept inputs for all these parameters, or will more accurately represent the Beer's Law absorption with a correct Poynting vector optical calculation.

The examples discussed in this paper utilize a generic quadrilayer MO film structure on a grooved substrate (see discussion of grooves below) as shown in Fig. 1. The nominal material thicknesses, as well as the base optical and thermal parameters employed, are listed in Table 1. The reflectivity of the nominal film stack in Fig. 1 is 0.22 at a wavelength of $660 \mathrm{~nm}$. The spot size parameter used is $\beta=(330 \mathrm{~nm})^{-1}$.

In thermal diffusion problems, one often is interested in characteristic diffusion lengths $L=(k t)^{0.5}$ or times $t=L^{2} / K$. The time resulting from $\kappa$ values from Table 1 and 
Table 1 Materials and their properties for a nominal quadrilayer MO structure (Fig. 1)

\begin{tabular}{|c|c|c|c|c|c|c|c|}
\hline Material & Thickness $(\mathrm{nm})$ & $\mathrm{n}$ & $\mathrm{k}_{\text {opt }}$ & $\mathrm{k}(\mathrm{W} / \mathrm{m}-\mathrm{K})$ & $\mathrm{c}(\mathrm{J} / \mathrm{Kg}-\mathrm{K})$ & $\rho\left(\mathrm{Kg} / \mathrm{m}^{3}\right)$ & $\mathrm{k}\left(10^{-6} \mathrm{~m}^{2} / \mathrm{s}\right)$ \\
\hline Air & $\infty$ & 1 & 0 & 0.03 & 1150 & 1.15 & 22.7 \\
\hline SiN & 85,15 & 2.02 & 0.01 & 2.0 & 740 & 3400 & 0.79 \\
\hline MO & 20 & 3.12 & 3.83 & 7.0 & 360 & 7900 & 2.46 \\
\hline AlX & 40 & 1.8 & 6.8 & 40,120 & 900 & 2700 & $16.5,49.4$ \\
\hline polycarbonate & $\infty$ & 1.58 & 0 & 0.15 & 300 & 1200 & 0.42 \\
\hline glass & $\infty$ & 1.5 & 0 & 1.0 & 860 & 2300 & 0.51 \\
\hline
\end{tabular}

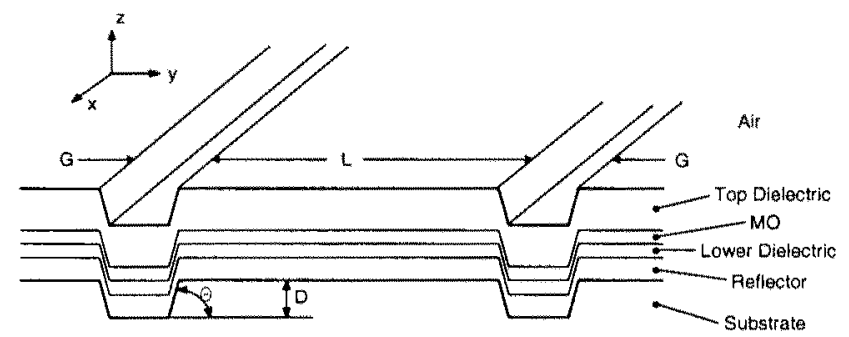

Fig. 1 Film stack geometry used in this work.

characteristics $L$ values for axial and lateral transport (given the geometry of the films and heating spot) clearly shows that lateral diffusion can be greatly diminished by exclusive use of short pulses for media heating ${ }^{4,5)}$. In Figures 2 and 3 we examine how light pulse energy deposited at a rate of $1 \mathrm{~mW}$ on a first-surface film stack

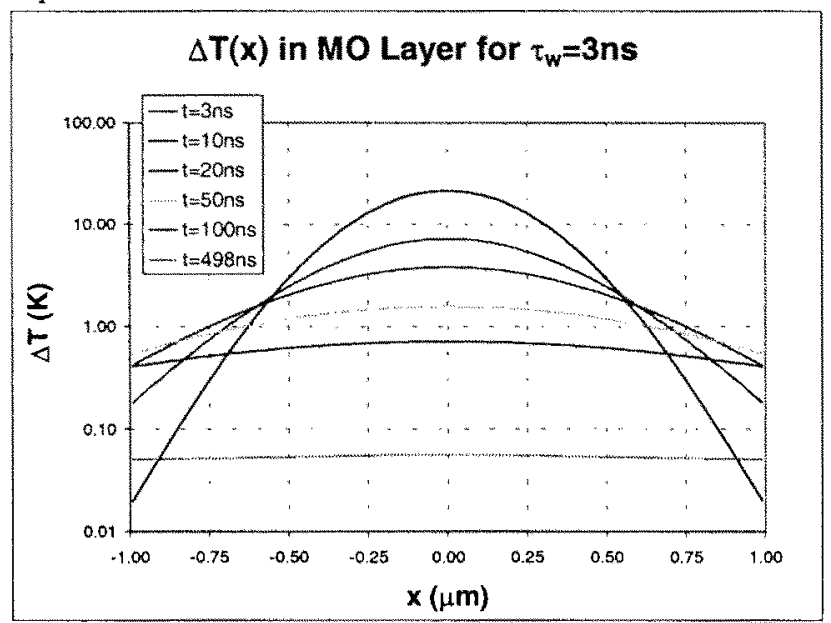

Fig. 2(a) Lateral heat diffusion for $\tau_{\text {pulse }}=3 \mathrm{~ns}$.

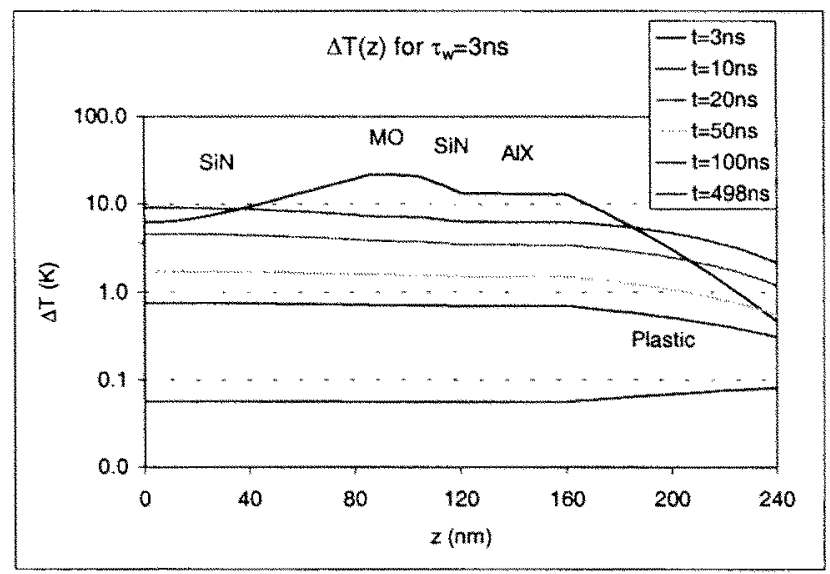

Fig. 2(b) Axial heat diffusion for $\tau_{\text {pulse }}=3$ ns.
(Fig. 1) from a static beam, but with either a short ( $3 \mathrm{~ns}$ ) or long $(-500 \mathrm{~ns})$ duration, diffuses both laterally in the film layer and axially through the layers. Figures 2(a) and 2(b) show the short duration heating case in which the absorbing MO layer heats up for $3 \mathrm{~ns}$, and then deposited heat diffuses away without further input. The relatively conductive MO layer spreads the heat laterally, while the AlX (a few atomic percent of dopant $X$ ) reflector / heat sink and massive substrate pull the heat downward axially. Figures 3(a) and 3(b) present the quite different result for the case of essentially continuous irradiation. The heating beam drives the peak temperature in the film stack (which occurs in MO film, the primary absorption layer) asymptotically upward, while the same diffusion processes observed in Figure 2 carry away heat. An examination of these two figures make it evident that a multiplicity of characteristic diffusion times and lengths

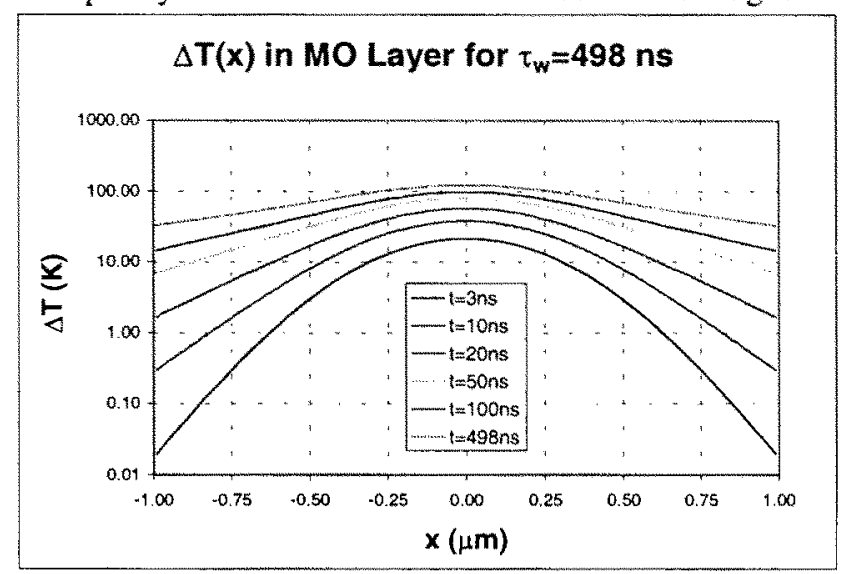

Fig. 3(a) Lateral heat diffusion for $\tau_{\text {pulse }}=498$ ns.

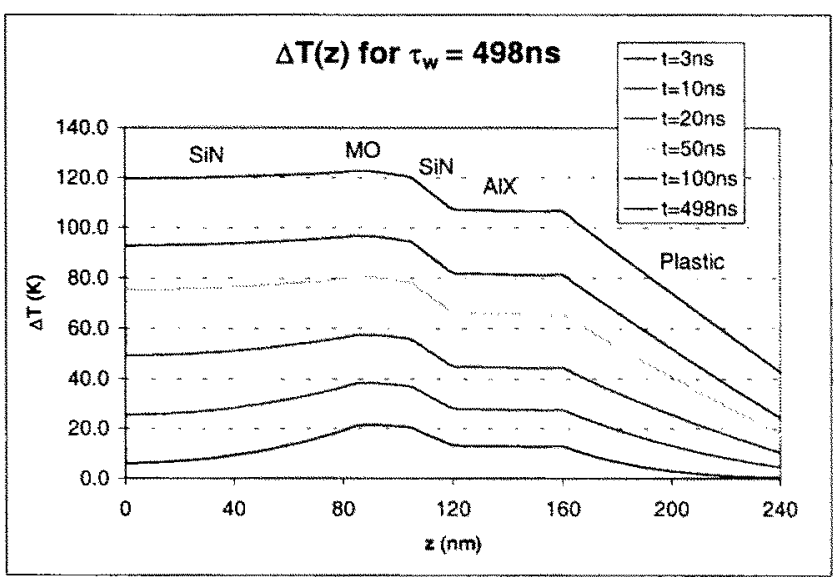

Fig. 3(b) Axial heat diffusion for $\tau_{\text {pulse }}=498 \mathrm{~ns}$. 
3),4) are present in a heterogeneous thin film stack (Fig. 1).

The essence of thin film thermal design in optical recording is adjusting the balance of multiple lateral and axial diffusion processes to achieve the desired recording function. This is obviously done through the choice of the film thicknesses and film material thermal properties, since thermal resistance conceptually is $R_{t h}=k A / l$, where $A$ and 1 are effective conductive path cross-section and length, respectively. It is important to note that the thermal constants of the films shown in Table 1 may be adjusted considerably by altering the film compositions or deposition processes ${ }^{4)}$. The role of relative velocity between the heating beam and the disk is overlaid on these fundamental diffusion processes, further spreading the energy input along the track direction. We will examine these velocity effects in more detail in later sections.

\section{Increasing Severity of Design Constraints}

One of the central issues of optical media thermal design has always been the search for ways of implementing the control of lateral heat flow. In writing, one uses an optical stylus (focused laser beam) of finite size to deposit sufficient heat to impart a local physical change in the media that can later be reliably detected on readout. But this marking must be done with adequate resolution to avoid interference or disturbance of adjacent written features (cross-write or cross-erase).

Generally, writing resolution is governed by three physical effects: (1) The size of the optical stylus (parameter $\beta^{-1}$ ); (2) the competition between lateral and axial heat diffusion; and (3) the heat distribution effects of optical power dosage by a moving stylus. The designers of an optical data storage system usually specify (1) and (3) to support the overall device storage capacity and data rate targets, while the media designers must address (2), given the constraints of (1) and (3). This presents a difficulty for media design when the system areal density requirements rise faster than the required scaled increase in the inverse spot area $\beta^{2}$, because shorter wavelength laser development generally does not keep pace with market areal density demands. In addition, the system requirement of higher data rate puts further pressure on the need for in-track writing resolution, as it forces linear speeds higher and spreads laser dosage along the track.

There exist analogous thermal effects on the readout side of the optical data storage and retrieval process, although these are more subtle since the reading power is usually a factor of three (at least) lower than writing powers. The main thermal concerns on readout are (a) reversible reduction of the Kerr effect, (b) irreversible changes in the stored magnetization, and (c) distortion or enlargement of thermally-induced super-resolution masks due to poor thermal gradients or incorrect sensitivity.

With these demands, what alternatives do media designers have? Traditionally, one focuses on proper film stack thermal design through material choice, fabrication process, and thickness selection. This approach gives some degree of control on power sensitivity and the balance between axial and lateral heat spreading, although there are usually severe constraints and tradeoffs involved here (mainly laser power availability and the accommodation of optical, $\mathrm{MO}$, and magnetic design). A system-level strategy that further aids in heat flow control is the laser pulsing strategy, as noted earlier. Short, high power pulsing of input optical energy provides the best efficiency, and allows axial heat diffusion processes to optimally counteract detrimental (and slower) lateral ones.

\section{Heat Confinement Structure}

In this paper we present one example of another media design approach that appears to be effective in counteracting lateral heat flow to adjacent tracks. In Fig. 1 we show the geometry of relatively narrow inter-track heat confinement grooves, which can be designed to minimize the lateral spread of heat to tightly packed adjacent tracks. The use of inter-track grooves is well known in optical storage. Originally, grooves were used as guide features for the track-following servo of the ODD, with a land-to-groove width ratio of about $2: 1$, and the data recording done on the wider feature. Later, a land/groove structure with a L/G ratio of $\sim 1: 1$ and recording on both feature was adopted. If flying optical heads with head-disk spacings appropriate for near-field optical recording or hybrid optical-magnetic recording are used, then $50 \%$ duty cycle circumferential grooves may be problematic for head flying stability. Our interest here is in considering thermal confinement features (" $G$ ") with recording on land ("L") only in which $L / G \sim 4-10$. Thus, we seek narrow, but thermally isolating features $(G)$. The desired goals in designing such features are (1) minimizing the top width of the thermal isolation feature so as to minimize the topographical disruption for a flying slider, and (2) choosing feature geometry and covering film processes so as to minimize thermal conduction across the feature.

We have used a 3D finite element (FE) heat flow model and $\mathrm{MO}$ recording process simulations to explore this issue using certain settings of parameters $L, G, D$, and $\Theta$, as summarized in Table 2 . With regard to geometry, only the depth and wall slope angle of the grooves were varied. Film coatings were assumed to be perfectly conformal, meaning the $z$ thickness of each film is everywhere constant. The geometries of Cases (d) and (e) were identical, but the thermal conductivity of all the films on the groove wall was dropped to $0.1 \mathrm{~W} / \mathrm{m}-\mathrm{K}$ for Case (e) only. This was done to simulate a situation in which the imagined film deposition on the groove walls became very thin, and near discontinuity in film material occurred. The same parameter of inverse spot size $\beta$ reported earlier was used for these simulations.

Table 2 Groove parameters studied.

\begin{tabular}{|l|c|c|}
\hline $\begin{array}{l}\text { CASES (all have } \mathrm{L}=0.48 \mu \mathrm{m} \& \\
\mathrm{G}=0.12 \mu \mathrm{m})\end{array}$ & $\mathrm{D}(\mathrm{nm})$ & $\Theta(\mathrm{deg})$ \\
\hline (a) No groove & 0 & N/A \\
\hline (b) Shallow groove & 40 & 69.4 \\
\hline (c) Intermediate groove & 60 & 76.0 \\
\hline (d) Deep groove & 80 & 79.4 \\
\hline (e) Deep, insulating groove & 80 & 79.4 \\
\hline
\end{tabular}


From Table 2 we see that the groove wall angles that we have simulated are quite steep compared to those of conventional MO disk tracking grooves. The motivation for steep walls is clearly to (a) minimize the overhead of unusable disk surface area, and (b) to minimize the crosssectional area of the thermally conducting films deposited there. This latter effect is responsible for the observed (see below) increasing thermal isolation effect of Cases (b) to (d) in Table 2. To the extent that film deposition on groove walls is diminished below the conformal coating assumption of this modeling, the more like Case (e) the Cases (b)-(d) will be.

In the FE simulations for each of the groove geometries of Table 2, we calculated a thermal impulse response for a Gaussian beam power input of $1 \mathrm{~mW}$ focused on the track land center. The impulse response calculation was done by turning on a 3 ns duration power pulse at time zero. The impulse had a 1 ps rise and fall time. The time evolution of the impulse response thermal field in the 3D grooved track geometry was captured over the history of its decay for a time long enough (usually at least $150 \mathrm{~ns}$ ) to provide accurate superposition computations of recording simulations. Figures 4 (a) to 4 (d) show isotherms in the $y-z$ plane (perpendicular to the track direction) for various instants of selected impulse solutions. These plots clearly illustrate the effectiveness of a properly designed confinement groove geometry. Our study shows that deeper grooves with steeper walls are increasingly effective, and achievement of reduced intrinsic thermal conductivity of the film material on the wall is the most effective process for heat confinement.

\section{Thermal Characteristic Curves}

To investigate the effectiveness of thermal confinement grooves and to illustrate the combined effects of heat diffusion in the disk surface with the dynamics of distributed power dosage on a moving disk, we present in this section results from computed thermal characteristic curves $^{4)}$. Figures $5(\mathrm{a})$ and $5(\mathrm{~b})$ illustrate some limiting behaviors for the groove cases of Table 2 (Cases (a) and (e) - no grooves and $80 \mathrm{~nm}$ deep, low thermal conductivity grooves, respectively). The curves show the

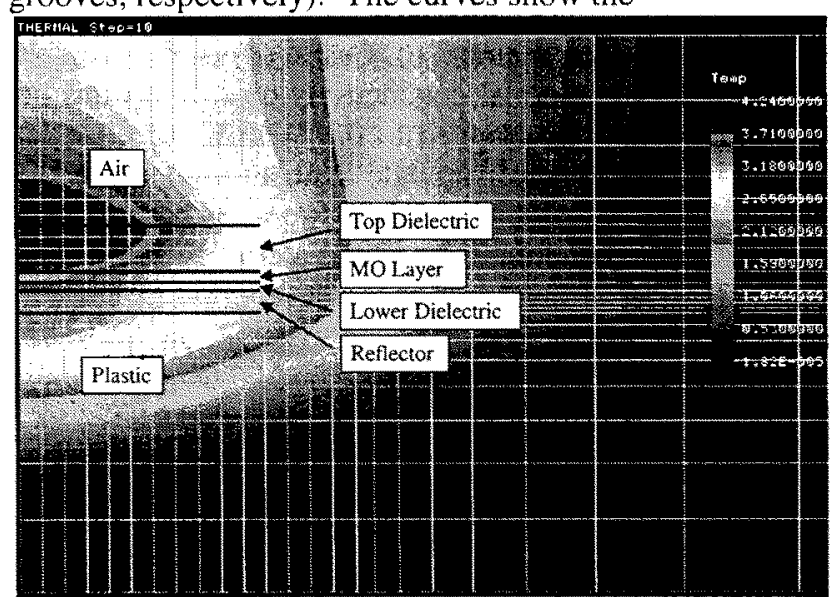

Fig. 4(a) $y-z$ plane isotherms of impulse solution for ungrooved media $(t=30 \mathrm{~ns})$.

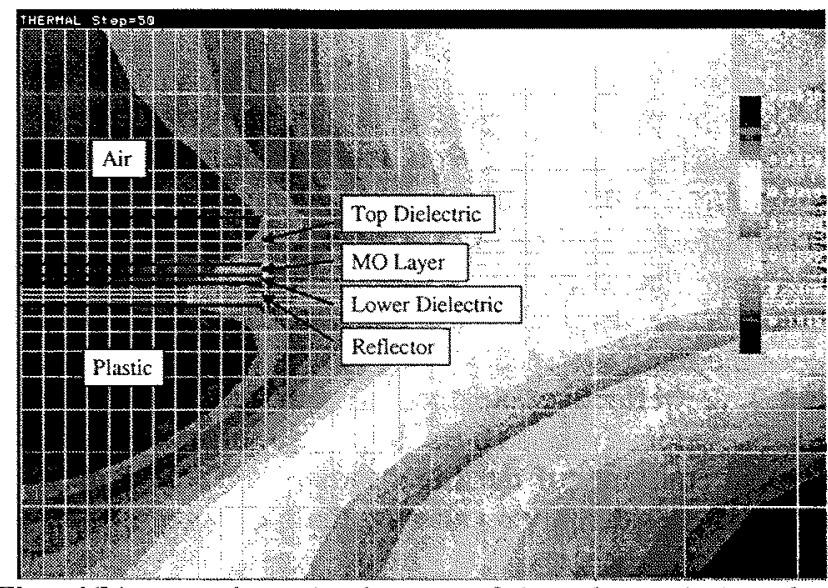

Fig. 4(b) y-z plane isotherms of impulse solution for ungrooved media $(t=150 \mathrm{~ns})$. Considerable lateral diffusion has occurred.

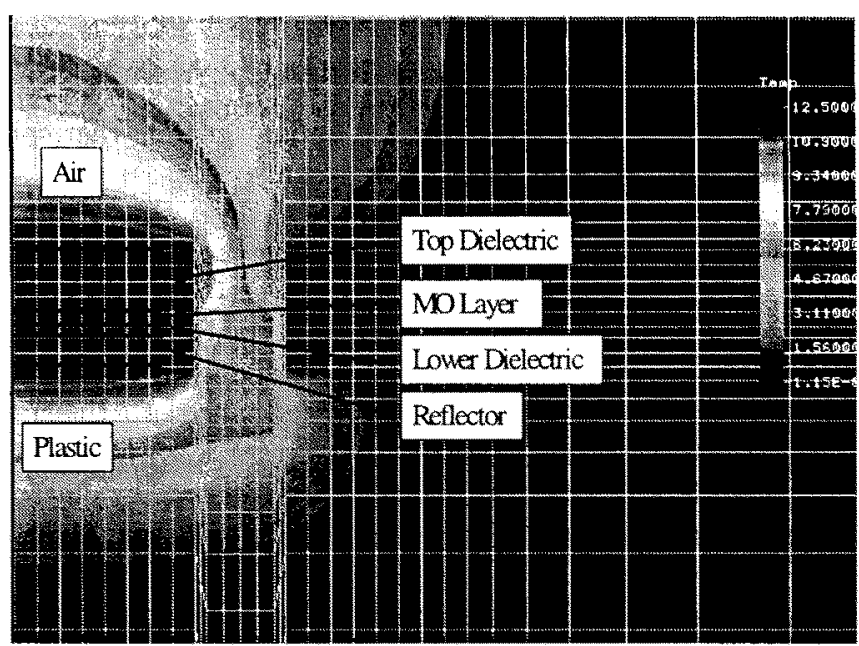

Fig. 4(c) y-z plane isotherms of impulse solution for media with $80 \mathrm{~nm}$ deep grooves of low thermal conductivity $(t=30$ ns $)$.

required isolated pulse power to form a $225 \mathrm{~K}$ temperature rise isotherm of cross-track width equal to $0.4 \mu \mathrm{m}$ or 0.8 $\mu \mathrm{m}$, that is, an estimate of the pulse power to write $\mathrm{MO}$

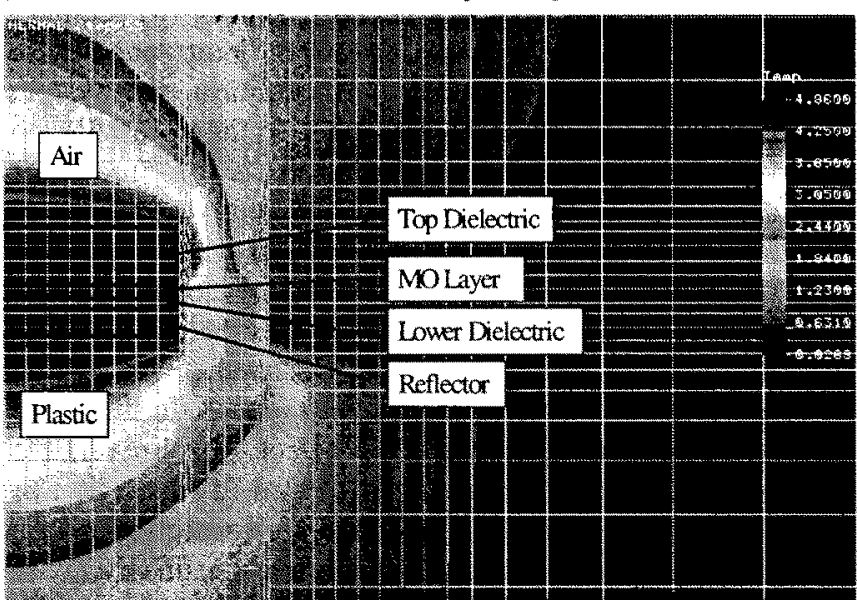

Fig. 4(d) $y-z$ plane isotherms of impulse solution for media with $80 \mathrm{~nm}$ deep grooves of low thermal conductivity $(t=150 \mathrm{~ns})$. Lateral diffusion is very effectively constrained. 


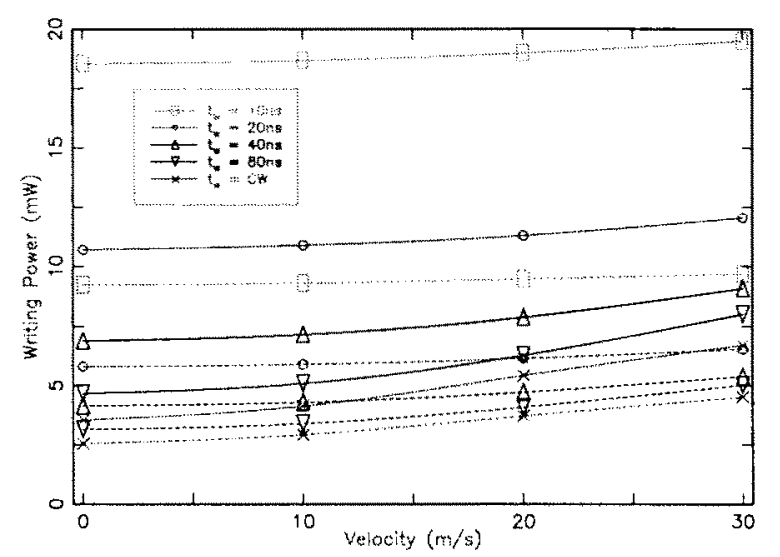

Fig. 5(a) Thermal characteristic curves for writing $0.4 \mu \mathrm{m}$ (dotted lines) and $0.8 \mu \mathrm{m}$ (solid lines) wide marks on flat, ungrooved media.

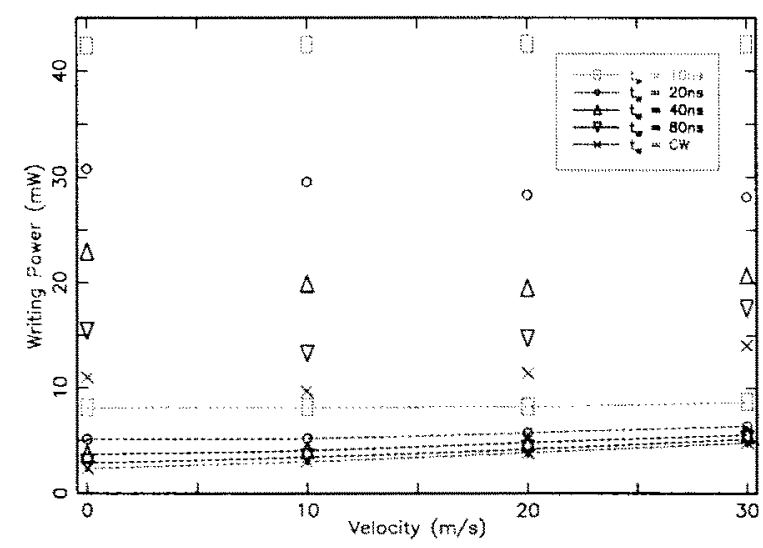

Fig. 5(b) Thermal characteristic curves for writing $0.4 \mu \mathrm{m}$ (dotted lines) and $0.8 \mu \mathrm{m}$ (points only) wide marks on media with $80 \mathrm{~nm}$ deep, low thermal conductivity grooves.

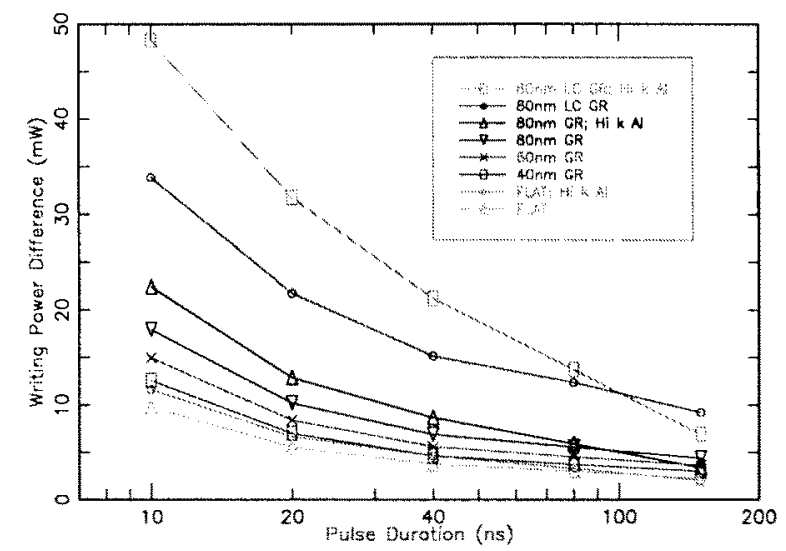

Fig. 6 Difference of writing powers for writing 0.8 and 0.4 $\mu \mathrm{m}$ wide marks at $\mathrm{v}=30 \mathrm{~m} / \mathrm{s}$ on all media.

marks of these widths. The curves cover ranges of writing pulse duration of $10 \mathrm{~ns}$ to $\mathrm{CW}$, and linear speeds from zero to $30 \mathrm{~m} / \mathrm{s}$. Because the heat from the writing pulse must cross the confinement groove to form the wider mark, a larger difference between the narrow and wide mark writing power is a measure of the effectiveness of the thermal groove. Figure 6 summarizes this data for all cases, and clarifies this point. This data reflects thermal effects only, and does not take into account magnetic domain wall dynamics effects in forming marks spanning a groove.

We also considered the effect of increasing the heat sinking effectiveness of the AIX layer by tripling its thermal conductivity (see Table 1). Characteristic curve data for this case is included in Fig. 6. In general, better axial heat sinking results in a flattening of thermal characteristic curves (lower velocity dependence). In Fig. 6 for the $0.8 \mu \mathrm{m}$ mark writing, there is evidence that a more conductive heat sink shunts heat under the groove barrier via the substrate for long pulse writing (see the curve cross-over for $\tau_{\mathrm{w}} \sim 100 \mathrm{~ns}$ ).

Two effects are very clear from Figs. 7 and 8 . While thermal confinement grooves can be quite effective in impeding cross-write or cross-erase, they have the inverse effect of greatly increasing the writing sensitivity ontrack. Note especially the low writing power required to write $0.4 \mu \mathrm{m}$ marks on the land of the low conductivity groove disk. To investigate how these thermal confinement effect impact the writing of high resolution information on-track, we simulated both light intensity modulation (LIM) and laser-pulsed magnetic field modulation (LP-MFM) writing on various of the disk designs of Table 2.

\section{Micromagnetic Simulations}

In comparing LIM and MFM writing, we should note that a clear consensus has developed as MO recording has evolved that MFM writing has several clear advantages for high density, high performance thermomagnetic recording. Chief among these are the relative absence of thermal domain edge shift effects in domain length modulation recording, provided that the media thermal environment at the site of recording is in constant phase relative to the data clock ${ }^{11)}$. Further, written domain lengths are not directly limited by the optical spot size as they are in LIM recording.

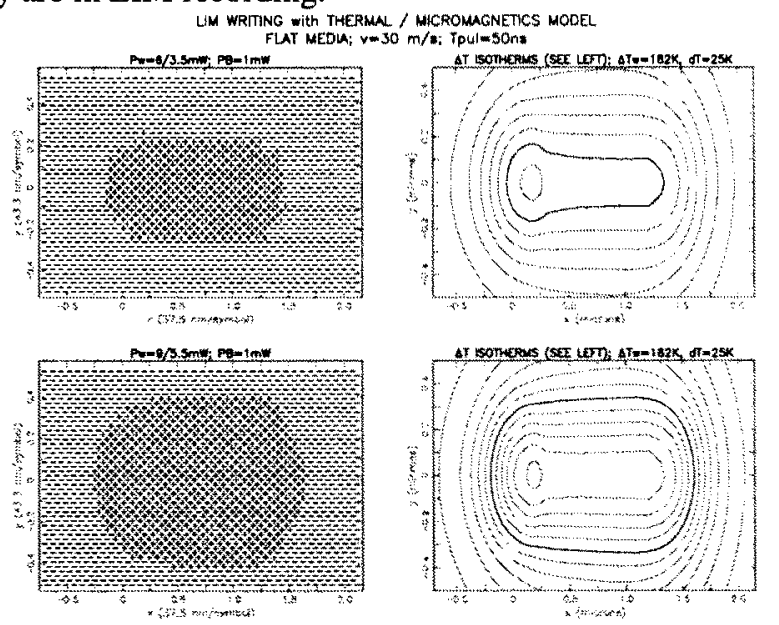

Fig. 7(a) LIM writing of a single domain on flat media (Case (a) of Table 2) at $\mathrm{v}=30 \mathrm{~m} / \mathrm{s}$ with two power levels. Notice that there is no impediment to mark widening. Modeled isotherms are shown for comparison. 


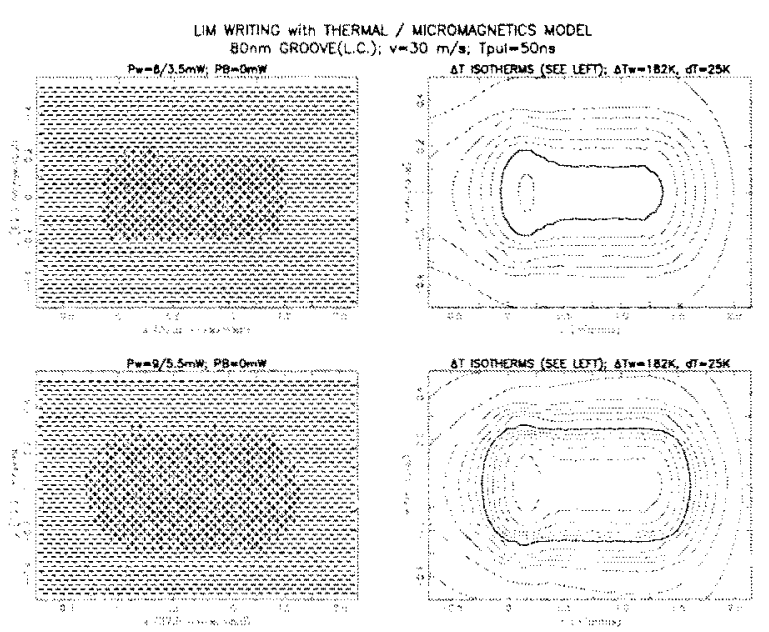

Fig. 7(b) LIM writing of a single domain on grooved, Case (e) media (Table 2) at $v=30 \mathrm{~m} / \mathrm{s}$ with two power levels. Notice that there is a strong impediment to mark widening due to the thermal confinement grooves. Modeled isotherms are shown for comparison.

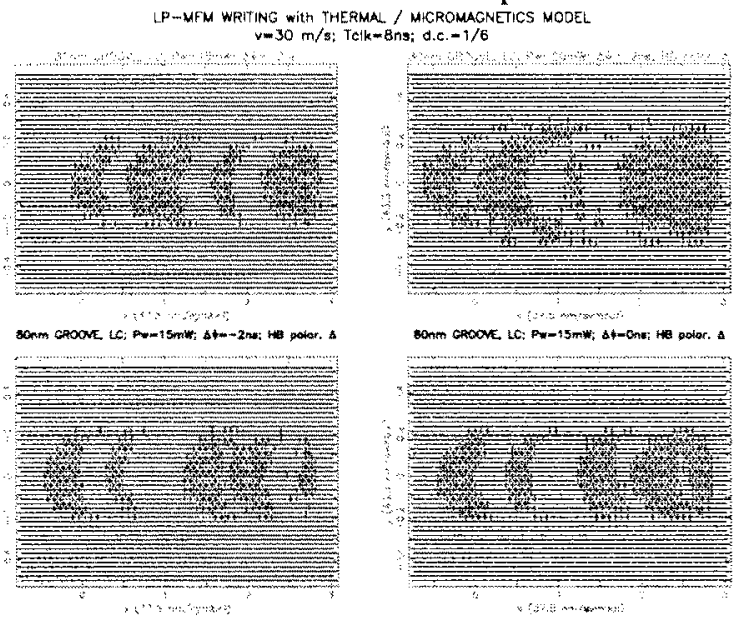

Fig. 8 An example of LP-MFM writing on grooved, Case (e) media (Table 2) at $v=30 \mathrm{~m} / \mathrm{s}$ simulated with the micromagnetics model. Upper left panel has reversed field polarity. The phase shift between the laser pulsing and field switching was also varied among the cases.

Even though LIM writing has been proven serviceable in existing $\mathrm{MO}$ products, recording simulations have shown that it is more difficult to implement with thermal confinement features that perform as illustrated in Fig. 6. Physically, confinement features parallel to the data tracks block radial heat flow and force blocked heat along the recording track. A LIM writing example in Figs. 7(a) and 7 (b) shows these effects. Notice how the lateral spread beyond the groove is greatly impeded. This makes accurate placement of domain walls in the $\pm x$ direction difficult, as radially blocked heat flows along the track.

We have simulated LP-MFM recording with a Monte Carlo micromagnetics model ${ }^{4), 12)}$ for media structures of Tables 1 and 2. Laser pulsed heating of the media was performed with a short power pulse on each data clock cycle. Pulse rise and fall times were set to $1 \mathrm{~ns}$, and pulse duty cycle was typcially $0.16-0.25$. Linear speeds covering $10-30 \mathrm{~m} / \mathrm{s}$ were simulated. Just as have previous workers ${ }^{11}$, we found that the best-formed crescent domains are written when the optimum phasing between the laser pulsing and field switching is determined.

In Fig. 8, we see examples of micromagnetically written crescent domains formed on Case (e) media from Table 2. The second panel shows the limited lateral spreading for a severely over-powered writing case, although the domains are poorly formed. An important result that we find for LP-MFM writing on thermal confinement media is that two thermal transport mechanisms combine to provide a desirable straightening of crescent edges across the recording track for reasonable writing power (see Figs. 7 (a) and 7(b)). Although down-track thermal gradients could be somewhat degraded by the confinement features, the gradients can be recovered with two familiar remedies: Low duty cycle laser pulsing (we used as low as 0.166 ) and increased axial heat sinking in the film structure.

\section{Conclusion}

A simulation study has determined that extension of well-known principles of MO media thermal design to regimes of higher speed and areal density can be effective when supplemented with additional steps. The use of intertrack thermal confinement features can protect against cross-write, while providing some advantage in shaping domains on-track.

\section{References}

1) See proceedings of 1998 Optical Data Storage Topical Meeting, 10-13 May, 1998, Aspen, CO U.S.A.; ISOM'98, 20-22 Oct., 1998, Tsukuba, Japan.

2) See papers by J. Davis at conferences in Ref. 1; Also, see all papers in Session We-I at ISOM'98 (Ref. 1).

3) H. Saga, etal: Paper Pd-08; and H. Nemoto, etal: Paper Pd-09, ISOM'98 Conference, Oct. 1998, Tsukuba, Japan.

4) T.W. McDaniel and R.H. Victora, eds.: Handbook of MO Data Recording, Chaps. 5,7,10 (Noyes Pubs., New Jersey, 1997).

5) D.S. Bloomberg and G.A.N. Connell: Chap. 6 of Magnetic Recording, vol. 3, C.D. Mee and E.D. Daniel, eds, (McGraw-Hill, 1988).

6) M. Mansuripur: The Physical Principles of MO

Recording, Chap. 11 (Cambridge Univ. Press, UK, 1995); M. Mansuripur, etal: Appl. Optics, 21, 1106 (1982); Appl. Optics, 22, 666 (1983); J. Appl. Phys., 54, 4794 (1983).

7) O.W. Shih: J. Appl. Phys., 75, 4382 (1994).

8) H.S. Carslaw and J.C. Jaeger: Conduction of Heat in Solids (Oxford Univ. Press, U.K. 1959).

9) A. Majumdar: Trans. ASME, J. Heat Transfer, 115, 7 (1993); M.I. Flik, B.I. Choi, and K.E. Goodson: Trans. ASME, J. Heat Transfer, 114, 666 (1992).

10) M.R. Madison and T.W. McDaniel: J. Appl. Phys., 66, 5738 (1989).

11) T. Niihara, etal: J. Magn. Magn. Mtls., 88, 177

(1990); S. Yonezawa and M. Takahashi: Appl. Optics, 33, 2333 (1994); Y. Tanaka, etal: Appl. Optics, 37, 2699 (1994)

12) T.W. McDaniel and M. Mansuripur: IEEE Trans. Magn., 23, 2943 (1987). 